\title{
Coronary revascularization after heart transplant - the search for prognostic factors
}

\author{
Bartlomiej Guzik ${ }^{1}$, Elżbieta Szczepanek ${ }^{1}$, Łukasz Niewiara ${ }^{1}$, Marcin Nosal ${ }^{1}$, Karol Wierzbicki², \\ Marcin Krzanowski ${ }^{3}$ Piotr Szolc ${ }^{1}$, Bogusław Kapelak², Krzysztof F. Żmudka ${ }^{1}$
}

\begin{abstract}
${ }^{1}$ Department of Interventional Cardiology, Institute of Cardiology, Jagiellonian University Medical College, John Paul II Hospital, Krakow, Poland

${ }^{2}$ Department of Cardiovascular Surgery and Transplantology, Institute of Cardiology, Jagiellonian University Medical College, John Paul II Hospital, Krakow, Poland

${ }^{3}$ Department of Nephrology, Jagiellonian University Medical College, Krakow, Poland
\end{abstract}

Submitted: 20 June 2017

Accepted: 8 November 2017

Arch Med Sci 2020; 16 (4): 789-795

DOI: https://doi.org/10.5114/aoms.2017.71847

Copyright (c) 2020 Termedia \& Banach

\begin{abstract}
Introduction: Survival after heart transplantation (HTX) is extended due to continuous improvement of medical care, allowing enough time for coronary artery vasculopathy to develop. Data on the clinical outcome of cardiac transplantation patients after percutaneous coronary intervention $(\mathrm{PCI})$ are still not extensively explored. The aim of our study was to assess whether heart transplantation itself compromises the outcome in patients undergoing percutaneous coronary intervention and to assess survival rates as well as major cardiovascular complications in heart transplant recipients who had undergone $\mathrm{PCl}$.
\end{abstract}

Material and methods: Thirty-three heart transplant recipients who had undergone PCl in the years 2005 to 2015 in a single center were matched by age, sex and main risk factors of arteriosclerosis with 33 controls without heart transplant history. Mean age of patients was $54.6 \pm 11.4$ years in the HTX group and $58.8 \pm 10.8$ years in controls. Median time from heart transplant to $\mathrm{PCl}$ was 13 years (4.4-22 years). Case and control groups did not differ in terms of standard risk factors of coronary artery disease, apart from chronic kidney disease, which was present in $70 \%$ of patients after heart transplantation, and dyslipidemia, which was present in $91 \%$ of control subjects. Results: Patients after HTX had worse survival compared to controls $(p=0.04)$. When adjusted for comorbidities in the Cox regression model, there was no significant difference in survival between cardiac transplant recipients and the control group $(\mathrm{HR}=1.06 ; 95 \% \mathrm{Cl}: 0.10-11.24)$. Chronic renal disease was a significant predictor of all-cause mortality $(H R=29.9$; 95\% Cl: 2.3-393). Considering other endpoints, HTX patients had considerably higher incidence of severe bleeding compared to the control group $(27 \%$ vs. $3 \%, p<0.05)$.

Conclusions: There was no significant difference in myocardial infarction rate, revascularization or hospitalization rates.

Key words: heart transplantation, percutaneous coronary intervention, survival analysis.

\section{Introduction}

Graft vasculopathy (GV) is one of the most important reasons for graft failure [1]. It is also a main reason for stenosis formation in graft epicar-
Corresponding author:

Bartlomiej Guzik

Department of

Interventional Cardiology

Institute of Cardiology

Jagiellonian University

Medical College

John Paul II Hospital

80 Prądnicka St

31-202 Krakow, Poland

E-mail: b.guzik@uj.edu.pl 
dial arteries, in contrast to non-transplant heart, where arteriosclerotic plaque is mainly responsible for myocardial ischemia [2]. The mechanisms of GV are increasingly understood. It is a combination of autoimmune response, cytomegalovirus (CMV) infection, ischemia-reperfusion and preservation damage. All these processes lead to vascular inflammation contributing to endothelial dysfunction that additionally affects donor-transmitted arteriosclerosis. This leads to transplant atherosclerosis [3]. From available studies, it is known that GV progresses and after 15 years since heart transplantation (HTX) significant GV is diagnosed among $40 \%$ of patients. This leads to higher mortality compared to GV free subjects. Percutaneous angioplasty improves this outcome [4].

Guidelines suggest performing coronary angiography even as frequently as once a year in patients after heart transplant $[5,6]$. As HTX patients are mostly angina free, coronary stenosis is sometimes a random finding and often accompanies the graft rejection process. The main pathomechanism of coronary stenosis in the graft is an autoimmunologic inflammatory response and infection causing vascular inflammation leading to endothelial dysfunction [7-10].

Having little data in the literature on the longterm follow-up in patients after HTX, to date we know that those patients benefit from percutaneous coronary intervention $(\mathrm{PCl})$ in the case of $\mathrm{GV}$. But we still do not know which factors influence outcomes. In the literature, there are limited available data showing predictors of higher mortality among those patients, but different comorbidities tend to progress after HTX [11]. Development of heart failure (ejection fraction $(\mathrm{EF})<40$ or systolic blood pressure (SBP) $<90 \mathrm{~mm} \mathrm{Hg}$ ) were independent survival predictors in HTX patients after $\mathrm{PCl}$. Chronic kidney disease was among the predictors but it was insignificant. Dyslipidemia treatment had a beneficial influence on those patients. Interventions on the right coronary artery (RCA) did not seem to influence outcomes, whilst $\mathrm{PCI}$ on other vessels was a significant predictor of survival $[4,12]$. It is interesting how HTX itself influences outcomes.

The aim of our study was to assess whether heart transplantation itself compromises the outcome in patients undergoing percutaneous coronary intervention and to assess survival rates as well as major cardiovascular complications in heart transplant recipients who had undergone $\mathrm{PCl}$.

\section{Material and methods}

We investigated 33 heart transplant recipients who underwent coronary angiography and $\mathrm{PCl}$ due to medical indications, in the Department of Inter- ventional Cardiology (Jagiellonian University, John Paul II Hospital), Krakow, Poland. Those patients were compared with other 33 patients without heart transplant history. This control group was matched by age, sex and main risk factors of coronary artery disease from a cohort of patients with stable coronary artery disease, after $\mathrm{PCl}$ but without a history of heart transplant.

This patient selection process was done by the head-to-head method. Matching between groups was based on age, arterial hypertension, diabetes (type 2, on oral or insulin therapy), obesity (body mass index $(\mathrm{BMI})>30 \mathrm{~kg} / \mathrm{m}^{2}$ ), prior acute coronary syndrome and target vessel. The head-to-head matching method was used, as propensity score matching involves regression analysis, which was planned for use in Cox models. Chronic kidney disease (stage 3 and 4) was more frequent in patients with HTX (vs. control; $p<0.001$ ). We found a major difference between groups in comorbidities of patients at the same age and the same occurrence of main risk factors (hypertension, diabetes, obesity, prior acute coronary syndrome (ACS)). Thus, propensity score matching was difficult to achieve. All patients underwent planned angiography due to medical indications.

We followed up patients in an out-patient clinic. Endpoints were assessed in all the patients during the follow-up. Data concerning ACS, stroke, and any cause of hospitalization were recorded and classified according to the ICD-10. CV endpoint was defined as occurrence of ACS, stroke and death due to these conditions. Deaths not caused by these conditions were classified as non-CV death. Composite endpoint (MACCE) was identified as a combination of CV endpoint with non-CV mortality, providing all-cause mortality. Bleeding was defined according to GUSTO bleeding criteria.

The study was a part of a statutory grant approved by the Jagiellonian University Ethics Committee.

\section{Statistical analysis}

Continuous variables were presented as a mean value with standard deviation or as a percentage for categorical values. Unless stated otherwise, comparison of means was performed with the two-sided $t$-test for variables with normal distribution and with the non-parametric Mann-Whitney $U$ test for distributions other than normal. The Kaplan-Meier estimator with log-rank test was used to assess survival of patients. Furthermore, multifactorial Cox regression analysis with adjustment for age, sex and comorbidities was applied to explore risk factors of death. In all analyses 0.05 was adopted as the level of significance. Statistical analyses were 
performed with SPSS ver. 23 (IBM, US) and R-Studio (RStudio 2016).

The propensity score matching method was planned to be used for the control group assignment. However, due to the low number of patients and low calculated propensity scores, and planned further regression analysis, the manual matching of controls was performed $[13,14]$.

The forest plot was prepared with $R$ statistical software [15]. For hazard ratio estimation of nonparametric parameters we plotted the hazard ratio curve for a continuous predictor using the SmoothHR R package [16].

\section{Results}

\section{Clinical characteristics}

Mean age of patients in the HTX group was $54.6 \pm 11.4$ compared to $58.8 \pm 10.8(p=N S)$ in the control group. Most of the patients were male. Median time from heart transplant to $\mathrm{PCl}$ was 13 years (4.4-22 years). Predominantly $\mathrm{PCl}$ was performed in the left anterior descending artery ( $42 \%$ cases), then in the right coronary artery (27\%) and the left circumflex artery (15\%). Matching the target vessels for revascularization between groups was $100 \%$.

The two groups did not differ significantly regarding risk factors, apart from dyslipidemia, which was more frequent in the control group (91\% vs. 48\%; $p<0.001$ ), and chronic kidney disease, which was present in over $60 \%$ of patients after heart transplantation (70\% vs. $6 \%$; $p<0.001)$. Details of comorbidity differences are tablepresented in Table I. The EF in both groups differed significantly (57\% vs. $44 \% ; p=0.02$ ) but none of the patients in the HTX group had EF below $40 \%$. In laboratory findings, the main differenc- es between the HTX and control group occurred in creatinine levels $(204 \pm 32$ vs. $105 \pm 12 \mathrm{mM}$; $p=0.006)$, estimated glomerular filtration rate (eGFR) as well as in hemoglobin levels (12.6 \pm 0.4 vs. $14.0 \pm 0.3 \mathrm{~g} / \mathrm{dl} ; p=0.01$ ). We also found that platelet counts were lower in the HTX group (187 \pm 8 vs. $241 \pm 11 ; 10^{3} / \mathrm{mm}^{3} ; p<0.001$ ); Table II summarizes differences in laboratory findings. There was also a significant difference in use of $\beta$-blockers ( $90 \%$ vs. $17 \% ; p<0.001$ ). That was the only considerable difference in concomitant treatment.

\section{Percutaneous intervention}

Patients in pairs had the same vessel revascularized. In the HTX group patients received more frequently drug-eluting stent (DES) than in the control group ( $97 \%$ vs. $79 \% ; p=0.026$ ). Multivessel revascularization was also more frequent in HTX patients ( $19 \%$ vs. $6 \% ; p=0.096)$. In 2 cases of revascularization in patients after HTX a bioresorbable scaffold was used. Otherwise angioplasty techniques did not differ significantly between patients regarding use of drug-eluting balloon (DEB), rotablation, cutting balloon, additional stents or balloons, or post-dilatation technique. There were no true bifurcations treated in either group. Dual antiplatelet therapy was applied according to guidelines and there was no difference in use of clopidogrel or aspirin between groups.

Patients after heart transplant were treated according to algorithms used in our center. Most patients were treated with cyclosporine, everolimus, tacrolimus, mycophenolate mofetil and steroids, which did not differ from the general HTX population of our center. Frequencies of the use of particular medications are presented in Table III. Between patients with chronic kidney disease

Table I. Characteristics and comorbidities in heart transplantation patients and control group

\begin{tabular}{|lccc|}
\hline Parameter & Post HTX $(n=33)$ & Control $(n=33)$ & $P\left(\chi^{2}\right)$ \\
\hline Female : male, $n: n$ & $7: 26$ & $8: 27$ & NS \\
\hline Age, mean (SD) [years] & $54.6(11.4)$ & $58.8(10.8)$ & NS* \\
\hline Time to HTX, median [years] & 13.0 & NA & NS \\
\hline Arterial hypertension, $n(\%)$ & $24(73)$ & $28(85)$ & $<0.001$ \\
\hline Dyslipidemia, $n(\%)$ & $16(48)$ & $30(91)$ & NS \\
\hline Diabetes type $2, n(\%)$ & $11(33)$ & $4(12)$ & NS \\
\hline Chronic kidney disease, $n(\%)$ & $23(70)$ & $2(6)$ & NS \\
\hline Obesity, $n(\%)$ & $2(6)$ & $10(21)$ & $0.02^{*}$ \\
\hline Prior ACS, $n$ (\%) & $13(39)$ & 44 & \\
\hline EF (\%) & 57 & $40)$ & \\
\hline
\end{tabular}

HTX - heart transplantation, ACS - acute coronary syndrome, NA - not applicable, EF-ejection fraction. ${ }^{*}$-test. 
Table II. Laboratory findings in heart transplantation patients and control group

\begin{tabular}{|lccc|}
\hline Parameter & Post HTX & Control & $P$-value* \\
\hline Total cholesterol, mean (SD) $[\mathrm{mmol} / \mathrm{l}]$ & $4.6(0.2)$ & $4.8(0.2)$ & $\mathrm{NS}$ \\
\hline LDL, mean (SD) $[\mathrm{mmol} / \mathrm{l}]$ & $2.7(0.2)$ & $3.1(0.2)$ & $\mathrm{NS}$ \\
\hline HDL, mean (SD) $[\mathrm{mmol} / \mathrm{l}]$ & $1.2(0.1)$ & $1.3(0.1)$ & $\mathrm{NS}$ \\
\hline TG, mean (SD) $[\mathrm{mmol} / \mathrm{l}]$ & $1.3(0.2)$ & $1.4(0.1)$ & $\mathrm{NS}$ \\
\hline Fasting glucose, mean $(\mathrm{SD})[\mathrm{mmol} / \mathrm{l}]$ & $6.2(0.3)$ & $6.4(0.4)$ & $\mathrm{NS}$ \\
\hline Creatinine, mean $(\mathrm{SD})[\mu \mathrm{mol} / \mathrm{l}]$ & $204.0(32.1)$ & $104.7(12.2)$ & 0.006 \\
\hline eGFR, mean $(\mathrm{SD})\left[\mathrm{ml} / \mathrm{min} / 1.73 \mathrm{~m}^{2}\right]$ & $46.4(4.6)$ & $68.4(5.9)$ & 0.006 \\
\hline WBC, mean (SD) $\left[10^{3} / \mu \mathrm{l}\right]$ & $7.9(0.7)$ & $8.1(0.5)$ & $\mathrm{NS}$ \\
\hline Hb, mean (SD) $[\mathrm{g} / \mathrm{dl}]$ & $12.6(0.4)$ & $14.0(0.3)$ & 0.01 \\
\hline HCT, mean $(\mathrm{SD})(\%)$ & $39.3(1.0)$ & $41.4(0.8)$ & $\mathrm{NS}$ \\
\hline PLT, mean $(\mathrm{SD})\left[10^{3} / \mu \mathrm{ll}\right]$ & $187.4(8.2)$ & $240.8(10.8)$ & $<0.001$ \\
\hline
\end{tabular}

HTX - heart transplantation. ${ }^{*}$-test.

Table III. Concomitant medications used by heart transplantation patients

\begin{tabular}{|lcccc|}
\hline Immunosuppressive therapy & Percentage of patients after HTX & \multicolumn{2}{c|}{ Chronic kidney disease } & \multirow{2}{*}{ P-value* } \\
\cline { 3 - 4 } & & No & Yes & \\
\hline Ciclosporin (\%) & 61 & 61 & 60 & 0.93 \\
\hline Everolimus (\%) & 44 & 38 & 50 & 0.51 \\
\hline Mycophenolate mofetil (\%) & 46 & 61 & 38 & 0.18 \\
\hline Steroids (\%) & 59 & 61 & 57 & 0.80 \\
\hline Tacrolimus (\%) & 27 & 23 & 29 & 0.72 \\
\hline
\end{tabular}

HTX - heart transplantation. ${ }^{*} P$-value $-\chi^{2}$ test.

Table IV. Endpoint comparison between groups

\begin{tabular}{|c|c|c|c|}
\hline Parameter & Post HTX & Control & $P$-value* \\
\hline All-cause mortality, $n(\%)$ & $16(48)$ & $2(6)$ & 0.001 \\
\hline MACCE, $n(\%)$ & $26(79)$ & $11(33)$ & 0.014 \\
\hline $\mathrm{ACS}, n(\%)$ & $4(12)$ & $4(12)$ & 0.745 \\
\hline TVR, $n(\%)$ & $7(21)$ & $7(21)$ & 0.651 \\
\hline Severe bleeding, $n(\%)$ & $9(27)$ & $1(3)$ & 0.018 \\
\hline Hospitalization, $n$ (\%) & $15(45)$ & $14(42)$ & 0.609 \\
\hline
\end{tabular}

$H T X$ - heart transplantation, MACCE - composite endpoint, ACS - acute coronary syndrome, TVR-total vessel revascularization. ${ }^{*} P$-value - $\chi^{2}$ test.

(CKD) and without there was no significant differences in immunosuppressive treatment. Also, complexity of immunosuppressive treatment (use of 3 or more medications) did not differ between these groups.

\section{Long-term follow-up}

Total time at risk was 127859 person-days. Median follow-up time was 1325 days (3.63 years; $255-4113$ days). The composite endpoint occurred in $49 \%$ of patients. All-cause mortality occurred in 18 patients (24\%); the majority of deaths occurred in men. Table IV summarizes the comparison of endpoints between groups.

There was a significant difference in severe bleeding between groups. We looked into this group of patients with severe bleeding. None of the risk factors differed significantly apart from gender and age. Females had severe bleeding more frequently 
than men ( $9 \%$ vs. $28 \% ; p=0.042)$. Those patients had a higher mortality rate than patients without bleeding. Exploratory logistic regression for severe bleeding as an endpoint did not show any factors that could predict severe bleeding occurrence.

\section{The search for prognostic factors}

Patients after heart transplantation had worse survival compared to controls, as presented in Figure 1 ( $p=0.04$ for Kaplan-Meier; log-rank test). When adjusted for age, gender, hypertension, dyslipidemia, diabetes and chronic kidney disease in the multivariable Cox- $\mathrm{PH}$ regression model, there was no significant difference in survival between cardiac transplant recipients and the control group. Chronic renal disease was a significant predictor of all-cause mortality ( $\mathrm{HR}=29.9 ; 95 \% \mathrm{Cl}: 2.3-393)$ as well as a protective effect of treatment of dyslipidemia ( $\mathrm{HR}=0.07 ; 95 \% \mathrm{Cl}: 0.01-0.44)$. Heart transplantation itself did not increase risk of mortality in this specific group $(\mathrm{HR}=1.06 ; 95 \% \mathrm{Cl}$ : 0.1-11.2). Figure 2 shows a forest plot presenting the results of Cox-PH regression. In addition, we calculated the continuous influence of eGFR on survival. Figure 3 shows eGFR hazard ratio (log) changes with confidence intervals depending on its values. The biggest fluctuations in slope can be observed between $50 \mathrm{ml} / \mathrm{min}$ and $80 \mathrm{ml} / \mathrm{min}$.

\section{Discussion}

The biggest study to date on long-term mortality of patients after heart transplant enrolled 90 patients after $\mathrm{PCl}$ [4]. In this study the authors compared patients after HTX and $\mathrm{PCl}$ to patients only after HTX. Coronary angioplasty did prolong life-span in those patients, compared to those with GV but not treated with $\mathrm{PCl}$. In our study, we chose to compare 66 patients after $\mathrm{PCl}$ : 33 HTX recipients vs. 33 non-HTX. We intended

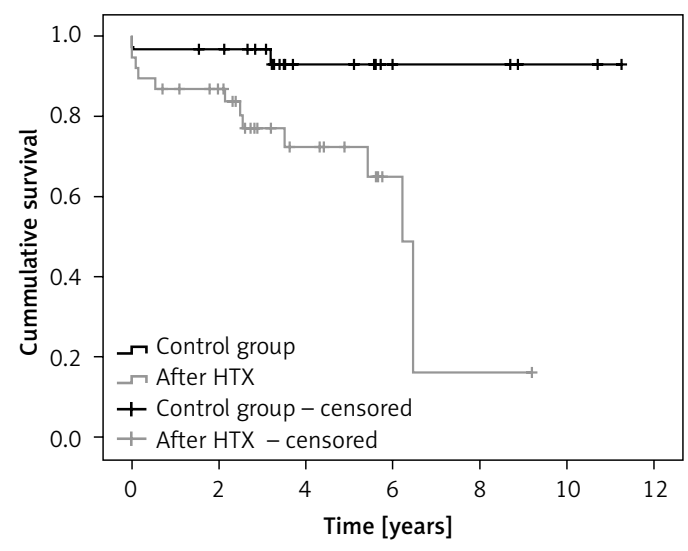

Figure 1. Overall survival in heart transplantation and control group (KM estimates, log-rank test)

that the only difference between patients in pairs would be HTX. In the literature, there is no comparison like this published.

In this study, we analyzed patients several years after the heart transplant when coronary vasculopathy developed causing significant narrowing requiring revascularization. In those patients, $\mathrm{PCl}$ prolongs life-span, as presented by Agarwal et al. [4].

In contemporary science, the propensity score matching method for control group assignment is gaining popularity, mainly due to its ability to decrease selection bias in case-control studies, similar to those observed in randomized control trials [14]. However, precise calculation of propensity scores and their relevant application are crucial for internal and external validity of the study [17]. In our population obtained propensity scores were low, probably due to the small number of patients. Therefore, manual matching of controls by age, sex, comorbidities and treated vessel was performed. Manual matching was very difficult due to common chronic kidney disease occurrence in the HTX group, which was hard

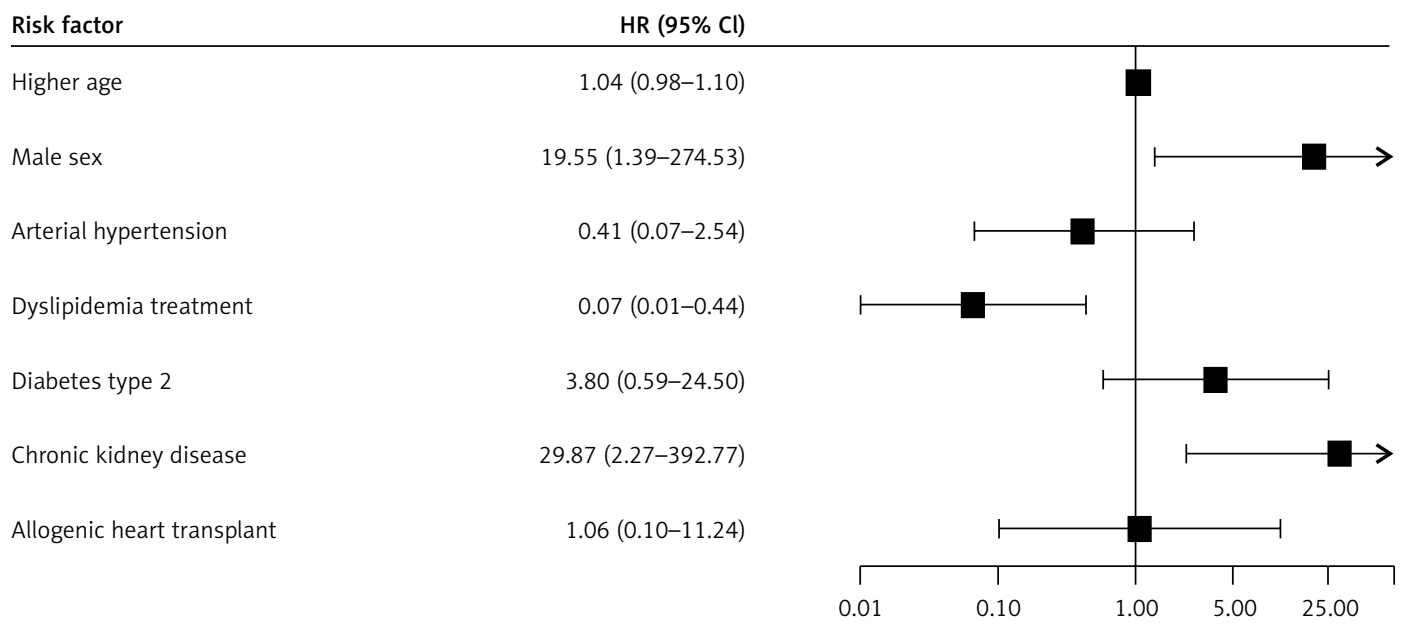

Figure 2. Risk factors of death in Cox regression model 


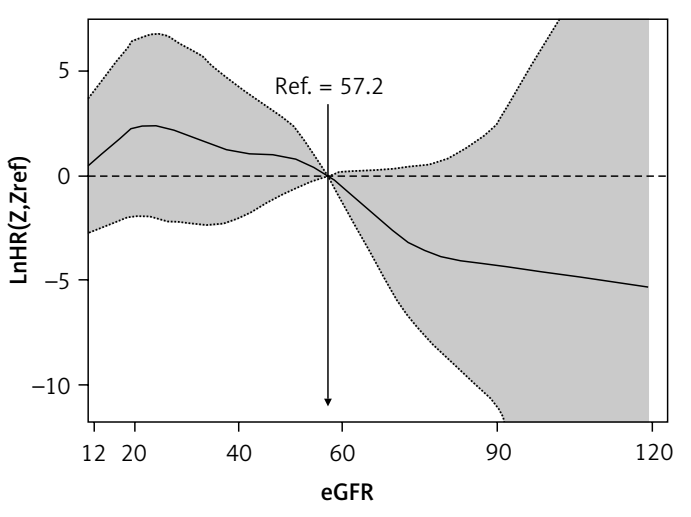

Figure 3. Hazard ratio (log) depending on estimated glomerular filtration rate (eGFR) value

to find in the control patient population. This could also be the additional reason for poor propensity matching. Arteriosclerosis plays a minimal role in developing stenosis. That is why we found dyslipidemia more often in the control group. There was a huge difference in use of $\beta$-blockers between groups. Patients after HTX have $\beta$-blockers introduced in our center when EF drops below $40 \%$ (none of the patients had decreased EF) or no bradyarrhythmia occurs in $24 \mathrm{~h}$ ECG monitoring.

Chronic kidney disease very often occurs in our patients requiring $\mathrm{PCl}$ as well as in those with chronic heart failure [18]. Atherosclerosis and vascular calcifications are the most common causes of increased mortality and morbidity in patients with CKD. Further analysis showed CKD to be a strong, independent predictor of mortality. Increased creatinine level is a known predictor of poor prognosis after decompensation of chronic heart failure [19]. Frequency of CKD after HTX is also caused by long use of immunosuppressive treatment, mainly calcineurin inhibitors (tacrolimus or cyclosporine). Patients after HTX have to use strong immunosuppressive treatment for lifetime, which causes several complications such as development of CKD. Patients developing CKD were shifted to treatment with everolimus.

In the studied group DES stents were used more frequently (compared to the control group). The literature suggests that DES stents do not bring an additional benefit in HTX patients [20].

When we looked at endpoints, there was a distinct difference in MACCE and all-cause mortality between groups. There was no difference in ACS and total vessel revascularization (TVR) between groups, which was expected to be a proof of unsuccessful revascularization. Also, no difference in hospitalization frequencies between groups suggests good out-patient care, especially in HTX patients. In the exploratory regression model, we did not find any predictive factors of severe bleeding. The reason for that could be sample size, as well as the fact that this was not the primary focus of this study but an accidental finding. This difference needs further exploration.

While searching for predictive factors of a significant difference of survival in the KM log-rank test it was surprising to find that undergoing heart transplant was not a predictive factor. Cox$\mathrm{PH}$ showed only CKD and dyslipidemia to play role in prediction of death. Propensity score calculations confirmed our findings. Statin use had a protective influence on survival, as shown previously in the literature $[4,20]$.

Wellnhofer et al. in their paper presented similar results to ours regarding survival but without a control group. Stenting did not improve graft survival [21]. They suggest that treatment with mycophenolate mofetil, and with concomitant treatment with statins, can have a beneficial effect on graft survival. In our population statins showed a protective effect, but introduction of mycophenolate mofetil into the Cox-PH model only increased the predictive value of CKD (up to $\mathrm{HR}=117.5 ; 95 \% \mathrm{Cl}: 3.6-3779)$. Use of mycophenolate mofetil was not significantly higher in patients with CKD. We did not adjust the model to the revascularized vessel since it was a crucial factor in patient matching. We also observed a protective effect of statin use in our patients. This was consistent with studies presented in the literature.

Since CKD was such a strong predictor of survival, we decided to look into whether HR changes depending on different values of eGFR. Figure 3 shows a plot of how HR (log) changes depending on the increase of eGFR. As expected, the higher the filtration rate, the less influence it has on survival. Of course, the confidence intervals are wide, but it is due to the small number of observations. But the significance of the CKD influence is so strong that it is unlikely to be an error.

Analyzing our data, we need to take the small sample size into consideration, especially when exploring subgroups of patients. These findings need some further analysis since, to our best knowledge, there are no papers on this subject. CKD is one of the most frequent complications after heart transplant and a recognized risk factor of graft failure [12, 22, 23].

There are two main limitations of this study. The first is the small sample size. In different statistics, we observed only trends instead of significant relationships, and the confidence intervals were very wide. Increasing the number of patients with $\mathrm{PCl}$ after HTX would probably solve the problem.

The second is heterogeneity of the study population. Almost every patient after HTX was unique, due to different duration and course of heart failure preceding HTX as well as comorbidity. 
In conclusion, patients after HTX compared to controls had a worse clinical outcome after $\mathrm{PCl}$. When adjusted for comorbidities in the Cox regression model, there was no significant difference in survival between cardiac transplant recipients and the control group. The difference in survival was caused by the presence of chronic kidney disease or diabetes, but not by the history of HTX. HTX patients more often suffered from severe bleeding compared to controls. A protective effect of statin use was observed. Further research in this specific population is needed to improve patient outcomes. There was no significant difference in myocardial infarction rate, revascularization or hospitalization rates.

\section{Conflict of interest}

The authors declare no conflict of interest.

\section{References}

1. Iyer $A$, Kumarasinghe $G$, Hicks $M$, et al. Primary graft failure after heart transplantation. J Transplant 2011; 2011: 175768.

2. Day J, Rayburn B, Gaudin P, et al. Cardiac allograft vasculopathy: the central pathogenetic role of ischemiainduced endothelial cell injury. J Heart Lung Transplant 1995; 14: S142-9.

3. Schmauss D, Weis M. Cardiac allograft vasculopathy recent developments. Circulation 2008; 117: 2131-41.

4. Agarwal S, Parashar A, Kapadia SR, et al. Long-term mortality after cardiac allograft vasculopathy: implications of percutaneous intervention. JACC Heart Fail 2014; 2: 281-8.

5. Ponikowski P, Voors AA, Anker SD, et al. 2016 ESC Guidelines for the diagnosis and treatment of acute and chronic heart failure: The task Force for the diagnosis and treatment of acute and chronic heart failure of the European Society of Cardiology (ESC)Developed with the special contribution of the Heart Failure Association (HFA) of the ESC. Eur Heart J 2016; 37: 2129-200.

6. Herre JM. Heart transplant medicine: beyond the guidelines. J Heart Lung Transplant 2013; 32: 1170-1.

7. Clerkin KJ, Ali ZA, Mancini DM. New developments for the detection and treatment of cardiac vasculopathy. Curr Opin Cardiol 2017; doi: 10.1097/HCO. 0000000000000388 [Epub ahead of print].

8. Schmauss D, Weis M. Cardiac allograft vasculopathy: recent developments. Circulation 2008; 117: 2131-41.

9. Guzik B, Chwała M, Matusik P, et al. Mechanisms of increased vascular superoxide production in human varicose veins. Pol Arch Med Wewn 2011; 121: 279-86.

10. Guzik TJ, Channon KM. Measurement of vascular reactive oxygen species production by chemiluminescence. Methods Mol Med 2005; 108: 73-89.

11. Wegrzyn P, Popiolek M, Przybylowski P, et al. The risk of cholelithiasis in patients after heart transplantation. Arch Med Sci 2014; 10: 53-7.

12. Hamour IM, Omar F, Lyster HS, Palmer A, Banner NR. Chronic kidney disease after heart transplantation. Nephrol Dial Transplant 2009; 24: 1655-62.

13. Wyss R, Girman CJ, LoCasale RJ, Brookhart AM, Stürmer T. Variable selection for propensity score models when esti- mating treatment effects on multiple outcomes: a simulation study. Pharmacoepidemiol Drug Saf 2013; 22: 77-85.

14. Austin PC, Mamdani MM. A comparison of propensity score methods: a case-study estimating the effectiveness of post-AMI statin use. Stat Med 2006; 25: 2084-106.

15. Gordon M. forestplot: Advanced Forest Plot Using 'grid' Graphics. 2016; Available from: https://cran.r-project. org/web/packages/forestplot/.

16. Meira-Machado L, Cadarso-Suárez C, Gude F, Araújo A. smoothHR: an $\mathrm{R}$ package for pointwise nonparametric estimation of hazard ratio curves of continuous predictors. Comput Math Methods Med 2013; 2013: 745742.

17. Austin PC. A critical appraisal of propensity-score matching in the medical literature between 1996 and 2003. Stat Med 2008; 27: 2037-49.

18. Franczyk-Skora B, Gluba A, Olszewski R, Banach $M$, Rysz J. Heart function disturbances in chronic kidney disease - echocardiographic indices. Arch Med Sci 2014; 10: 1109-16.

19. Feola M, Lombardo E, Testa M, Avogadri E, Piccolo S, Vado A. Prognostic factors of mid-term clinical outcome in congestive heart failure patients discharged after acute decompensation. Arch Med Sci 2012; 8: 462-70.

20. Park KIE, Huo T, Muller KE, Aranda JM, Hill JA, Anderson DR. Drug-eluting stents may not reduce target lesion revascularization in cardiac allograft vasculopathy. J Interv Cardiol 2014; 27: 80-5.

21. Wellnhofer E, Hiemann NE, Hug J, et al. A decade of percutaneous coronary interventions in cardiac transplant recipients: a monocentric study in 160 patients. J Heart Lung Transplant 2008; 27: 17-25.

22. Parikh PB, Jeremias A, Naidu SS, et al. Impact of severity of renal dysfunction on determinants of in-hospital mortality among patients undergoing percutaneous coronary intervention. Catheter Cardiovasc Interv 2012; 80: 352-7.

23. Thomas HL, Banner NR, Murphy CL, et al.; Steering Group of the UK Cardiothoracic Transplant Audit. Incidence, determinants, and outcome of chronic kidney disease after adult heart transplantation in the United Kingdom. Transplantation 2012; 93: 1151-7. 